\title{
Filter life span in postoperative cardiovascular surgery patients requiring continuous renal replacement therapy, using a postdilution regional citrate anticoagulation continuous hemofiltration circuit
}

\author{
Agnieszka Kośka ${ }^{1}$, Christopher J. Kirwan ${ }^{2}$, Maciej M. Kowalik ${ }^{3}$, \\ Anna Lango-Maziarz ${ }^{4}$, Wiktor Szymanowicz ${ }^{1}$, Dariusz Jagielak ${ }^{5}$, Romuald Lango ${ }^{3}$ \\ ${ }^{1}$ Department of Cardiac Anesthesiology, University Clinical Center, Gdansk, Poland \\ ${ }^{2}$ Department of Adult Critical Care, Royal London Hospital, Barts Health NHS Trust, \\ London, United Kingdom \\ ${ }^{3}$ Department of Cardiac Anesthesiology, Medical University of Gdansk, Poland \\ ${ }^{4}$ Department of Gastroenterology and Hepatology, Medical University of Gdansk, Poland \\ ${ }^{5}$ Department of Cardiovascular Surgery, Medical University of Gdansk, Poland
}

\begin{abstract}
Background: Regional citrate anticoagulation $(R C A)$ is the recommended standard for continuous renal replacement therapy (CRRT). This study assesses its efficacy in patients admitted to critical care following cardiovascular surgery and the influence of standard antithrombotic agents routinely used in this specific group.

Methods: Consecutive cardiovascular surgery patients treated with postdilution hemofiltration with $R C A$ were included in this prospective observational study. The primary outcome of the study was CRRT circuit life-span adjusted for reasons other than clotting. The secondary outcome evaluated the influence of standard antithrombotic agents (acetylsalicylic acid [ASA], low molecular weight heparin [LMWH] or fondaparinux as thromboprophylaxis or treatment dose with or without ASA) on filter life. Results: Fifty-two patients underwent 193 sessions of continous veno-venous hemofiltration, after exclusion of 15 sessions where unfractionated heparin was administered. The median filter life span was 58 hours. Filter life span was significantly longer in patients receiving therapeutic dose of LMWH or fondaparinux (79 $h$ [2-110]), in comparison to patients treated with prophylactic dose of LMWH or fondaparinux (51 $h$ [7-117], $p<0.001$ ), and patients without antithrombotic prophylaxis $(42 \mathrm{~h}$ [2-91], $p<0.0001) .12$ bleeding episodes were observed; 8 occurred in patients receiving treatment dose anticoagulation, 3 in patients receiving prophylactic dose anticoagulation and 1 in a patient with no antithrombotic prophylaxis.

Conclusions: A postdilution hemofiltration with RCA provides prolonged filter life span when adjusted for reasons other than clotting. Patients receiving treatment dose anticoagulation had a significantly longer filter life span than those who were on prophylactic doses or ASA alone. (Cardiol J 2022; 29, 1: 53-61)
\end{abstract}

Key words: cardiovascular surgical procedures, continuous renal replacement therapy, hemofiltration, anticoagulants, citric acid

Address for correspondence: Romuald Lango, MD, PhD, Department of Cardiac Anesthesiology, Medical University of Gdansk, ul. Dębinki 7, 80-211 Gdańsk, Poland, tel: +48 5858442 06, fax: +48 5858442 10, e-mail: rlango@gumed.edu.pl 


\section{Introduction}

Acute kidney injury (AKI) requiring continuous renal replacement therapy (CRRT) after cardiac surgery affects between $2 \%$ and $5 \%$ of patients and carries a mortality rate between $36 \%$ and $78 \%[1,2]$. Regional citrate anticoagulation (RCA) is the Kidney Disease-Improving Global Outcome (KDIGO) recommended anticoagulation of choice for CRRT and is of particular value to patients with contraindications to heparin and high risk of bleeding such as those who have undergone cardiac surgery [3, 4]. RCA is also associated with a prolonged filter life-span due to a decreased incidence of clotting and preservation of the filter sieving coefficient for larger molecules including inflammatory mediators $[5,6]$. This anticoagulation modality was reported to be safe and preferred after cardiac surgery [7].

Therefore, although RCA is commonly used in continuous veno-venous hemodialysis (CVVHD) and hemodiafiltration (CVVHDF) modes as these potentially offer reduced risk of clotting over purely convective modes, continuous veno-venous hemofiltration $(\mathrm{CVVH})$ with RCA on a high cut-off membrane may be of specific benefit to this group of patients provided filter life span remains long $[8,9]$. The Nikkiso Aquarius CRRT platform has been proven to be simple, effective and safe in delivering RCA for a postdilution CVVH method with a calcium containing replacement fluid in a general critical care population [10].

Cardiac surgery patients often need postoperative antithrombotic prophylaxis but the type and dose varies. Most coronary surgery patients require antiplatelet therapy, while patients after valve surgery can be treated with bridge antithrombotic prophylaxis with unfractionated heparin (UFH) or low molecular weight heparin (LMWH), until they can reach therapeutic international normalized ratio (INR) values resulting from oral anticoagulants administration though routine use remains open for debate [11].

As RCA has been reported to improve filter survival in patients receiving systemic anticoagulation with UFH for indications other than CRRT, e.g. patients on veno-arterial extracorporeal membrane oxygenation, it is the default anticoagulation method for CRRT in post cardiac surgery patients, in the absence of contraindications to citrate [12].

This study aims to evaluate the effect of postdilution CVVH with RCA anticoagulation on filter life span in general in this specific group of patients, and whether routine thromboprophylaxis adds additional benefit.

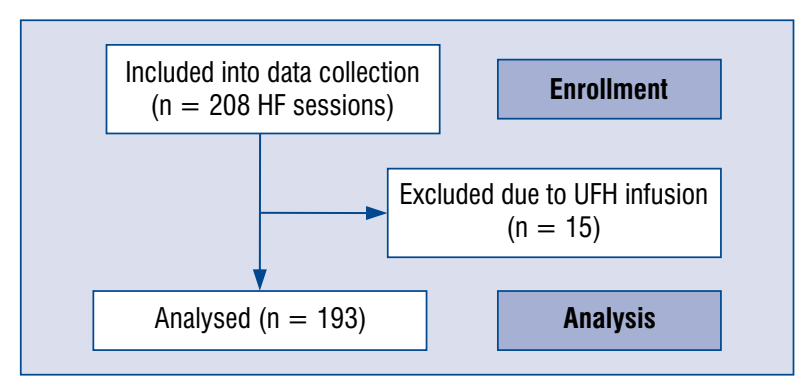

Figure 1. The flow-chart of hemofiltration sessions included into continuous renal replacement therapy circuit lifespan analysis; HF - heart failure; UFH - unfractionated heparin.

\section{Methods}

The study protocol conformed to the Ethical Principles for Medical Research Involving Human Subjects outlined in the Declaration of Helsinki, was approved by the local institutional review board for scientific studies (NKBBN/539/2016-17) and is registered in the Clinical Trials database (NCT03836742). Consecutive cardiovascular surgery patients treated with CVVH RCA between September 2015 and November 2017 were included (Fig. 1).

The decision to initiate renal replacement therapy was made by the treating clinician based on clinical features of volume overload and biochemical features of azotemia [3]. Contraindications to RCA were severe chronic liver disease, acute liver injury with INR $>2$ and refractory shock with lactate increasing above $8 \mathrm{mmol} / \mathrm{L}$.

\section{Hemofiltration and RCA protocol}

Continuous veno-venous hemofiltration was performed with the Aquarius + CRRT machine with version 6.02.14/15 software (Aquarius system, NIKKISO Europe GmbH, Desbrocksriede 1, 30855 Langenhagen, Germany) and CITRASET RCA for therapies with regional citrate anticoagulation comprising Aqualine RCA (Haemotronic S.p.A, Via Carreri 16, 41037 Mirandola, Italy) and AQUAMAX hemofilter (Nikkiso Belgium bvba, Industriepark 6, 3300 Tienen, Belgium). Filter size (either Aquamax $1.2 \mathrm{~m}^{2}$ or Aquamax $1.9 \mathrm{~m}^{2}$ ) was determined by the treating clinician and depended on the patient's actual body weight $(>90 \mathrm{~kg}$ ) and or the presence of distributive shock requiring noradrenalin infusion over $0.1 \mu \mathrm{g} / \mathrm{kg} / \mathrm{h}$, where an Aquamax 19 was used. In all patients Accusol 35 K0 (Nikkiso, Belgium Industriepark 6 B-3300 Tienen; Belgium), which contains $1.75 \mathrm{mmol} / \mathrm{L}$ of Calcium, was used as 
the postdilution replacement fluid. Anticoagulant citrate dextrose solution A, U.S.P. (ACD-A) (ACD-A, Macopharma, 5003F Rue Lorthiois, 59420 Mouvaux, France) was used as the source of citrate.

Three thousand units of UFH were added to $1 \mathrm{~L} 0.9 \% \mathrm{NaCl}$ solution for CRRT circuit priming in all but 7 patients who had a suspicion of heparin induced thrombocytopenia (HIT).

Initial settings of hemofiltration parameters and its modifications when metabolic alkalosis was observed, were adopted from the CVVH RCA protocol published by Kirwan et al. [10], however the renal dose was calculated for the actual body weight.

The plasma concentration of ionized calcium (iCa) was augmented with additional calcium supplementation initially using a dose of $10 \mathrm{~mL}$ of $10 \%$ Calcium Chloride (WZF Polfa, Karolkowa Str. 22/24, Warsaw, Poland) added to $1 \mathrm{~L}$ of normal saline which resulted in $\mathrm{Ca}^{++}$concentration of $4.6 \mathrm{mmol} / \mathrm{L}$. Due to low ionized calcium plasma concentration, after the first 10 patients its dose was increased to $20 \mathrm{~mL} 10 \%$ calcium chloride, and after the following 10 patients to $40 \mathrm{~mL} 10 \%$ calcium chloride, equivalent to $\mathrm{Ca}^{++}$concentration of $18.4 \mathrm{mmol} / \mathrm{L}$. The target calcium concentration in plasma was increased from the original 0.9-1.2 range to a 1.0-1.2 range. Similarly, the original protocol was modified by an additional routine infusion of $0.2 \mathrm{~g} / \mathrm{h}$ magnesium sulfate.

Blood flow was set to achieve a filtration ratio of $\sim 20 \%$. If the plasma $\mathrm{pH}$ increased above 7.5 or bicarbonate concentration above $40 \mathrm{mmol} / \mathrm{L}$ the hemofiltration dose was decreased from the initial $35 \mathrm{~mL} / \mathrm{kg} / \mathrm{h}$ to $25 \mathrm{~mL} / \mathrm{kg} / \mathrm{h}$. If metabolic alkalosis persisted, the citrate dose was reduced and if this did not resolve the issue within $3 \mathrm{~h}$ RCA was stopped [10].

Either 13.5 Fr Mahurkar catheters (Covidien, 15 Hampshire Str., Mansfield, MA 02048USA) or 12 Fr Hemo-Access (Biometrix Ltd., 4 Kiryat Mada, Jerusalem, Israel) with length depending on cannulation site were used, however the type of the catheter was not reported in patients' files.

Primary endpoint of this study was to assess filter lifespan in postdilution CVVH with RCA in cardiovascular surgery patients. Secondary endpoint evaluated the difference in filter life span depending on standard antithrombotic agents by separating hemofiltration sessions into three groups; Group A: no antithrombotic medication or acetylsalicylic acid (ASA) alone; Group B: prophylactic dose LMWH or fondaparinux, with or without ASA; Group C: treatment dose LMWH or fondaparinux with or without ASA. Fondaparinux was used instead of LMWH whenever HIT was suspected and HIT ELISA test was positive [13].

Treatment sessions where continuous UFH added for therapeutic reasons were excluded from filter survival analysis.

\section{Statistical analysis}

Data are presented as median, quartiles and ranges. CRRT circuit lifespan is presented as Kaplan-Meier curves. Categorical variables were compared between groups with the $\chi^{2}$ test. Intergroup differences in filter lifespan were assessed with the Cox-Mantel test.

\section{Results}

Two hundred and eight CVVH sessions were performed in 52 patients (including 4 chronic dialysis patients). Fifteen sessions were excluded as the patients received an additional UFH infusion. Patients characteristics and cardio-vascular surgical procedures are presented in Table 1.

\section{Primary endpoint}

Filter life span of 193 sessions was adjusted for reasons to stop the filter other than clotting (96) and these included patient death (3), clinical decision (14), transport to computed tomography scan (2), change of therapy (2), technical (3: 1 alkalosis, 1 machine failure, 1 access concern) and end of filter life span (73).

Median time CRRT circuit lifespan was $58 \mathrm{~h}$ (2-117). CRRT circuit lifespan reached the 72-h manufacturer cut off in 75 (38.9\%) sessions (Fig. 2).

\section{Secondary endpoint}

A significantly longer filter life span was observed in RCA CVVH sessions when patients received a treatment dose LMWH or fondaparinux (Table 2, Fig. 3).

Bleeding complications were observed in 12 patients (23\%) (6 major) during or after CRRT and are presented in Table 3. Nine patients with bleeding complications were treated with treatment dose LMWH or fondaparinux, one with UFH, one with prophylactic dose LMWH and one with ASA alone (NS).

\section{Discussion}

According to available research, this is the first study of postdilution CVVH with RCA antico- 
Table 1. Patient characteristics and type of surgical procedures in the study group.

\begin{tabular}{|c|c|}
\hline Patient characteristics/type of surgery & Data/number of patients \\
\hline Patients age & $\begin{array}{c}70\left(\mathrm{Q}_{1}=62, \mathrm{Q}_{3}=74 ;\right. \\
\text { range: } 37-84)\end{array}$ \\
\hline Sex (male) & 28 \\
\hline Preexisting renal disease & 24 \\
\hline ESRF/chronic dialysis treatment & 4 \\
\hline Diabetes mellitus & 12 \\
\hline Arterial hypertension & 34 \\
\hline COPD & 4 \\
\hline Hyperthyroidism & 3 \\
\hline Hypothyroidism & 3 \\
\hline Chronic atrial fibrillation & 11 \\
\hline Preoperative hemoglobin $<10[\mathrm{~g} / \mathrm{dL}]$ & 16 \\
\hline Preoperative creatinine concentration [mg/dL] & $\begin{array}{c}1.49\left(\mathrm{Q}_{1}=1.05, \mathrm{Q}_{3}=2.11 ;\right. \\
\text { range: } 0.62-6.18)\end{array}$ \\
\hline \multicolumn{2}{|l|}{ Type of surgery: } \\
\hline Valvular surgery & 23 \\
\hline Revascularization surgery (CABG and OPCABG) & 7 \\
\hline Valvular + revascularization & 6 \\
\hline Cardiac other & 2 \\
\hline Vascular surgery including thoracic aorta surgery & 10 \\
\hline Extracorporeal support (ECMO/VAD) & 1 \\
\hline Heart transplant & 1 \\
\hline Pericardial drainage & 1 \\
\hline PM electrodes removal & 1 \\
\hline Hospital mortality & $29(57 \%)$ \\
\hline
\end{tabular}

CABG - coronary artery bypass graft; COPD — chronic obstructive pulmonary disease; ECMO - extracorporeal membrane oxygenation; ESRF — end-stage renal failure; PM — pacemaker; OPCABG — off-pump coronary artery bypass graft; VAD — ventricular assist device

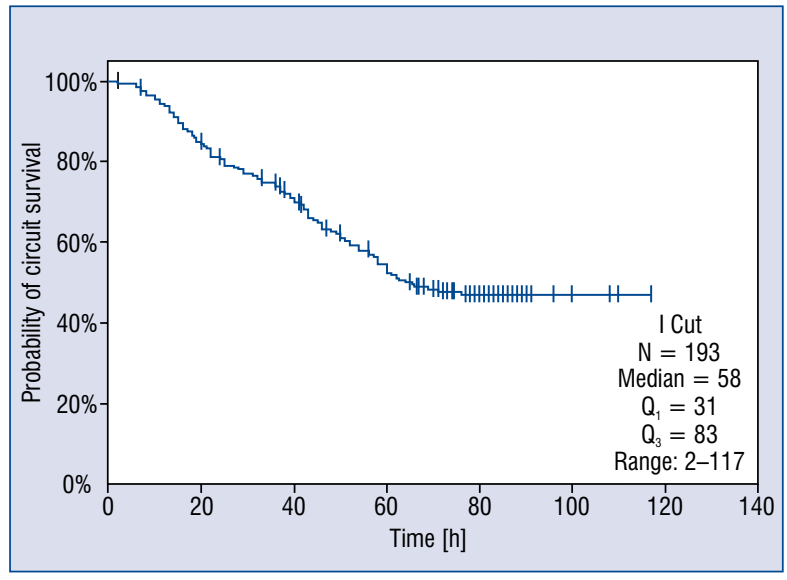

Figure 2. Kaplan-Meier curve of filter survival censored for discontinuation due to reasons other than circuit clotting. agulation in postoperative cardiovascular surgery patients. It is safe and effective treatment leading to a median filter life of $58 \mathrm{~h}$ when adjusted for reasons other than clotting.

The present results compare well with similar studies, using RCA in a general intensive care unit (ICU) population $(27 \mathrm{~h}$ ) and postoperative cardiac surgical patients who received RCA predilution continuous hemofiltration ( $48 \mathrm{~h})$, continuous hemodiafiltration with RCA or heparin (50-58 h) and continuous hemodialysis with RCA (39-61 h) [10, 14-20]. Most of these studies, however, did not specifically address the question of whether the patients received any systemic antithrombotic prophylaxis.

Although this is only a pilot study it is the first to demonstrate significant differences in RCA 
Table 2. Systemic antithrombotic prophylaxis used during regional citrate anticoagulation continuous veno-venous hemofiltration sessions.

\begin{tabular}{|c|c|c|}
\hline Antithrombotic agents & $\begin{array}{c}\text { Number of } \\
\text { hemofiltration } \\
\text { sessions }\end{array}$ & $\begin{array}{l}\text { Median circuit } \\
\text { lifespan hours } \\
\text { (range) }\end{array}$ \\
\hline Group A (n = 56) & & $42(2-91)$ \\
\hline No antithrombotic medication & 51 & \\
\hline ASA alone & 5 & \\
\hline Group B $(n=62)$ & & $51(7-117)$ \\
\hline Prophylactic dose low molecular weight heparin (LMWH) without ASA & 29 & \\
\hline Prophylactic dose LMWH with ASA & 16 & \\
\hline Prophylactic dose fondaparinux without ASA & 17 & \\
\hline Prophylactic dose fondaparinux with ASA & 0 & \\
\hline \multicolumn{3}{|l|}{ Group C ( $n=75)$} \\
\hline Treatment dose LMWH without ASA & 44 & $79(2-110)$ \\
\hline Treatment dose LMWH with ASA & 26 & \\
\hline Treatment dose fondaparinux without ASA & 5 & \\
\hline Treatment dose fondaparinux with ASA & 0 & \\
\hline
\end{tabular}

ASA — acetylsalicylic acid; LMWH — low molecular weight heparin

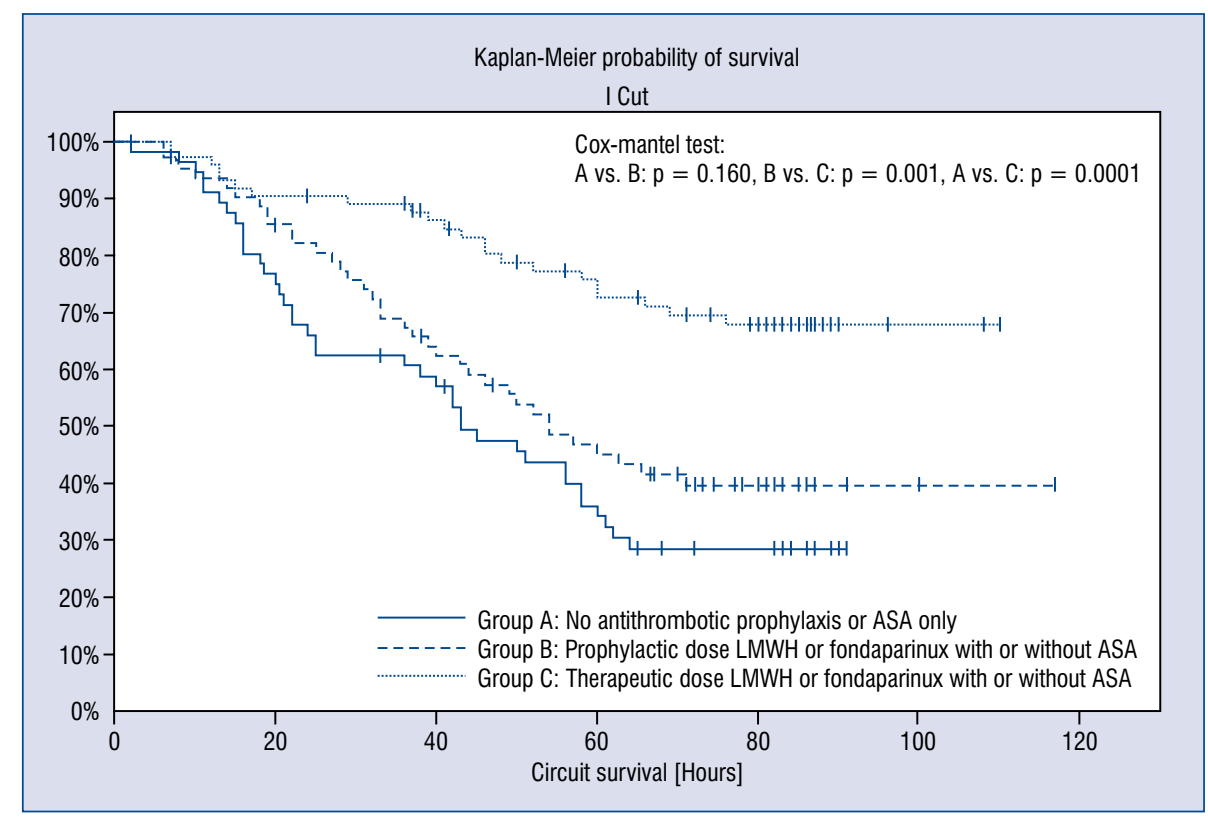

Figure 3. Influence of systemic antithrombotic prophylaxis on continuous renal replacement therapy (CRRT) circuit lifespan. (Group A: No antithrombotic prophylaxis or acetylsalicylic acid [ASA] only, Group B: prophylactic dose low molecular weight heparin [LMWH] or fondaparinux with or without ASA, Group C: more than prophylactic dose LMWH or fondaparinux with or without ASA).

CVVH filter lifespan between patients receiving additional systemic anticoagulation medications that are often required following cardiovascular surgery. This data is in line with a similar study of general ICU patients by Wu et al. [21] where the addition of low dose dalteparin to high volume predilution
CVVH with RCA improved filter life span from 25 to $40 \mathrm{~h}$, without increasing the risk of bleeding, and thus plays an important role when evaluating the success of specific filter anticoagulation or when comparing filter life span in cardiac patients and general ICU populations [21]. 
Table 3. Bleeding complications in the study group. Information on systemic antithrombotic prophylaxis refers to the highest dose during the whole hemofiltration treatment.

\begin{tabular}{|c|c|c|c|c|}
\hline Type of bleeding & $\begin{array}{l}\text { Surgical } \\
\text { procedure }\end{array}$ & $\begin{array}{c}\text { Systemic antithrombotic } \\
\text { prophylaxis }\end{array}$ & Remarks & Survival \\
\hline $\begin{array}{l}\text { Hematoma of ascending } \\
\text { colon mesentery }\end{array}$ & AVR, MVR & LMWH therapeutic dose & & No \\
\hline Airway bleeding (minor) & $\begin{array}{l}\text { Cardiac sarcoma } \\
\text { resection }\end{array}$ & LMWH therapeutic dose & $\begin{array}{l}\text { Idiopathic thrombocyto- } \\
\text { penia before surgery }\end{array}$ & No \\
\hline Airway bleeding (minor) & CABG & $\begin{array}{l}\text { Fondaparinux } \\
\text { prophylactic dose }\end{array}$ & HIT suspected & No \\
\hline $\begin{array}{l}\text { Retroperitoneal } \\
\text { hematoma }\end{array}$ & $\begin{array}{l}\text { MVR, TVP, ASD- } \\
\text {-closure, CABG }\end{array}$ & $\begin{array}{l}\text { Fondaparinux } \\
\text { therapeutic dose }\end{array}$ & $\begin{array}{l}\text { Suspected relation } \\
\text { to dialysis catheter } \\
\text { inserted through } \\
\text { femoral vein }\end{array}$ & No \\
\hline $\begin{array}{l}\text { Mediastinal hematoma, } \\
\text { gastro-duodenal bleeding }\end{array}$ & AVR & No prophylaxis or ASA only & & No \\
\hline Airway bleeding (minor) & TVP & LMWH therapeutic dose & & No \\
\hline $\begin{array}{l}\text { Airway bleeding and oral } \\
\text { cavity mucosal bleeding } \\
\text { (minor) }\end{array}$ & OPCABG & $\begin{array}{c}\text { LMWH therapeutic dose, } \\
\text { at } 3^{\text {rd }} \text { session replaced with } \\
\text { fondaparinux prophylactic } \\
\text { dose }\end{array}$ & HIT suspected & No \\
\hline Airway bleeding (minor) & MVR & LMWH therapeutic dose & Thrombocytopenia & No \\
\hline $\begin{array}{l}\text { Retroperitoneal bleeding in } \\
\text { the iliopsoas muscle area }\end{array}$ & AVR & LMWH therapeutic dose & $\begin{array}{l}\text { Suspected relation } \\
\text { to dialysis catheter } \\
\text { inserted through } \\
\text { femoral vein }\end{array}$ & Yes \\
\hline $\begin{array}{l}\text { Oral cavity mucosal } \\
\text { bleeding (minor) }\end{array}$ & AVR, CABG & LMWH therapeutic dose & & No \\
\hline $\begin{array}{l}\text { Femoral hematoma after } \\
\text { IABP removal }\end{array}$ & VA ECMO, IABP & UFH infusion & $\begin{array}{l}\text { Myocarditis was an } \\
\text { indication to VA ECMO }\end{array}$ & Yes \\
\hline $\begin{array}{l}\text { Retroperitoneal bleeding in } \\
\text { the iliac muscle area } \\
6 \text { days after the end of } \\
\text { hemofiltration treatment }\end{array}$ & MVR & LMWH therapeutic dose & $\begin{array}{l}\text { HIT, endocarditis was } \\
\text { an indication to MVR, } \\
\text { bleeding } 6 \text { days after the } \\
\text { end of hemofiltration. } \\
\text { Suspected relation to } \\
\text { dialysis catheter inserted } \\
\text { through femoral vein }\end{array}$ & Yes \\
\hline
\end{tabular}

ASA - acetylsalicylic acid; ASD — atrial septal defect; AVR — aortic valve replacement; CABG — coronary artery bypass graft; HIT — heparin induced thrombocytopenia; IABP - intra aortic balloon pump; LMWH - low molecular weight heparin; MVR - mitral valve replacement; OPCABG - off-pump coronary artery bypass graft; TVP — tricuspid valve plastic; UFH — unfractionated heparin; VAD - ventricular assist device; VA ECMO - veno-arterial extracorporeal membrane oxygenation

The incidence of bleeding is an important marker of treatment safety during CRRT. RCA reduces bleeding complications but many postoperative cardiac patients receive additional anticoagulants [11]. In the current study, major and minor bleeding complications observed in 8 patients were related to treatment dose anticoagulation. High incidence of bleeding complications observed in the present group of patients treated with CRRT was unlikely to be related to CRRT circuit anticoagulation but to post cardiovascular surgery antithrombotic prophylaxis. It is a matter of ongoing discussion if LMWH or UFH bridging is indispensable after valve surgery [11].
An appropriately powered study to assess bleeding complications when prophylactic LMWH or fondaparinux is added, is needed before this can be recommended as a routine addition to RCA CRRT therapy to increase filter survival alone and this will be difficult to do. A limitation of the current study is that we assessed hemofiltration sessions during which different antithrombotic prophylaxis were compared. In some patients different antithrombotic regimens were used depending on their clinical status. For example, if the mechanical mitral valve replacement patient was treated with CRRT in the early postoperative hours due to anuria, no antithrombotic prophylaxis was used due to 
the risk of bleeding. The same patient would however receive treatment dose of LMWH a few days later, often after the chest drains were removed. A more complex statistical comparison of bleeding episodes between the groups would be needed to evaluate the risk of bleeding post operatively, as well as a more formalized assessment of bleeding risk depending on time after surgery.

There are some clear limitations to this pilot study. It was not possible to capture data on catheter type and tip position, something which has been shown to play an important role in filter survival regardless of anticoagulation method [22].

Similarly, other factors potentially affecting circuit life span including platelet count and other measures of clotting function (e.g. analysis of thromboelastography) were not collected. Future studies of filter life span would benefit from including these laboratory data to determine if they are relevant in the clinical setting.

The life span of hemofiltration circuit can also be affected by multiple clinical factors including type of surgery, duration of surgery and cardiopulmonary bypass, aortic cross-clamp time, use of vasopressors, etc. A much larger study would be needed to individually evaluate these and it may not be of overall benefit.

There is however an additional potential benefit of demonstrating a good filter life span outcome with CVVH. A recent study revealed that after cardiac surgery postoperative inflammatory response is severe enough to fulfill systemic inflammatory response syndrome criteria in as many as $28 \%$ of patients [23]. Data from experimental and clinical studies, suggest that convection might be more effective than diffusion in the clearance of middle- and high-molecular weight particles at the equivalent dose, but the clinical benefit of convection above dialysis has not been shown [24].

In the original Royal London Hospital RCA protocol, a lower target citrate concentration $(2.8 \mathrm{mmol} / \mathrm{L})$ in the filter was accepted, in comparison to most RCA protocols for hemodialysis. ACD-A citrate solution was used instead of trisodium citrate solution, most commonly used throughout Europe, in order to reduce the risk of metabolic alkalosis resulting from citrate metabolism, and to decrease sodium load. It was presumed that target citrate concentration in the filter equal to $2.8 \mathrm{mmol} / \mathrm{L}$ should decrease ionized calcium concentration in the filter to $0.35 \mathrm{mmol} / \mathrm{L}$ on the average.

Some modifications were made to the original Royal London CVVH with RCA treatment formula.
Firstly, actual body weight instead of ideal body weight was used for renal dose calculation. The rationale for it was that patients after recent cardiovascular surgery have significant catabolism, and the authors intended to use a higher clearance, rather than too low of a clearance of solutes.

Secondly, the target plasma calcium concentration was higher in comparison to the original protocol. In order to achieve this goal, calcium concentration in calcium replacement solution was stepwise increased after initial experience, from $4.6 \mathrm{mmol} / \mathrm{L}$ to a final $18.4 \mathrm{mmol} / \mathrm{L}$. Higher range of target calcium concentration could potentially slightly increase the risk of filter clotting, because with a higher plasma ionized calcium concentration and equal dose of citrate, ionized calcium concentration in the filter could also be slightly increased in comparison to the observations of the authors of the original protocol. A target plasma ionized calcium concentration was increased in order to minimize the risk of hypocalcemia, which could contribute to exacerbating post cardiotomy heart failure and cause calcium resorption from bones during prolonged treatment of patients immobilized by their critical condition.

Hight cut-off ( $55 \mathrm{kDa}$ ) filters used in the study patients might theoretically promote convective cytokine removal, but its efficacy was not specifically studied in post cardiac surgery patients with AKI. This effect may be beneficial in the early postoperative hours after cardiopulmonary bypass cardiac surgery where high concentrations of cytokines and chemokines can play a significant role in contributing to increased postoperative morbidity and mortality and using the present protocol may be a way, in future, of evaluating its potential benefit $[25,26]$. To add further impetus in performing this type of study, there is some experimental rationale to preference RCA when initiating CRRT for postoperative cardiac patients. $\mathrm{RCA}$ is related to less complement and neutrophil activation in comparison to heparin, which may be an important factor in cardiac surgery patients, in whom complement and neutrophil activation by cardiopulmonary bypass, contributes to postoperative complications [27]. Citrate has been shown to exert cardio- and reno-protective effects in AKI triggered by ischemia-reperfusion in rats but this is yet to be shown in the clinical setting [28]. An improvement of survival in critically ill surgical patients treated with RCA in comparison to LMWH was observed by Oudemans-Van Straaten but it was not confirmed by subsequent studies [4, 29]. 


\section{Conclusions}

In summary, postdilution CVVH RCA for postoperative cardiovascular surgery patients results in excellent filter life span when compared to data from other groups of similar patients. The addition of treatment dose $\mathrm{LMWH} /$ fondaparinux required for post cardiac surgery antithrombotic prophylaxis significantly increases filter life span, but may carry a risk of increased incidence of bleeding episodes. Further studies are needed to determine whether routine additional anticoagulation is beneficial and whether a CVVH postdilution RCA protocol, that affords long filter life spans, can add additional mortality and morbidity benefit to postoperative cardiac surgical patients with renal failure over other modes of CRRT.

\section{Acknowledgments}

The authors would like to express their thanks to physicians and nurses in the Department of Cardiac and Vascular Surgery for their support in this study.

Conflict of interest: Christopher Kirwan: speaker fees from Baxter and Nikkiso and unrestricted educational grant from Nikkiso. Romuald Lango: speaker fees from Nikkiso and Fresenius Medical Care.

\section{References}

1. Pickering JW, James MT, Palmer SC. Acute kidney injury and prognosis after cardiopulmonary bypass: a meta-analysis of cohort studies. Am J Kidney Dis. 2015; 65(2): 283-293, doi: 10.1053/j.ajkd.2014.09.008, indexed in Pubmed: 25445101.

2. Chew ST, Hwang NC. Acute kidney injury after cardiac surgery: a narrative review of the literature. J Cardiothorac Vasc Anesth. 2019; 33(4): 1122-1138, doi: 10.1053/j.jvca.2018.08.003, indexed in Pubmed: 30228051.

3. Kidney Disease Improving Global Outcome (KDIGO) Acute Kidney Injury Work Group: KDIGO Clinical Practice Guidelines for Acute Kidney Injury. Kidney Int. 2012; 2(Suppl.): 1-138.

4. Bai M, Zhou M, He L, et al. Citrate versus heparin anticoagulation for continuous renal replacement therapy: an updated metaanalysis of RCTs. Intensive Care Med. 2015; 41(12): 2098-2110, doi: 10.1007/s00134-015-4099-0, indexed in Pubmed: 26482411.

5. Siebeck M, Dimski T, Brandenburger T, et al. Super High-Flux Continuous Venovenous Hemodialysis Using Regional Citrate Anticoagulation: Long-Term Stability of Middle Molecule Clearance. Ther Apher Dial. 2018; 22(4): 355-364, doi: 10.1111/17449987.12656, indexed in Pubmed: 29417731.

6. Liu C, Mao Z, Kang H, et al. Regional citrate versus heparin anticoagulation for continuous renal replacement therapy in critically ill patients: a meta-analysis with trial sequential analysis of randomized controlled trials. Crit Care. 2016; 20(1): 144, doi: 10.1186/s13054-016-1299-0, indexed in Pubmed: 27176622.
7. Borisov A, Malov A, Kolesnikov S, et al. Renal replacement therapy in adult patients after cardiac surgery. J Cardiothorac Vasc Anesth. 2019; 33(8): 2273-2286, doi: 10.1053/j.jvca.2019.02.023.

8. Oudemans-van Straaten HM, Ostermann M. Bench-to-bedside review: Citrate for continuous renal replacement therapy, from science to practice. Crit Care. 2012; 16(6): 249, doi: 10.1186/ cc11645, indexed in Pubmed: 23216871.

9. Friedrich JO, Wald R, Bagshaw SM, et al. Hemofiltration compared to hemodialysis for acute kidney injury: systematic review and meta-analysis. Crit Care. 2012; 16(4): R146, doi: 10.1186/ cc11458, indexed in Pubmed: 22867021.

10. Kirwan CJ, Hutchison R, Ghabina S, et al. Implementation of a simplified regional citrate anticoagulation protocol for postdilution continuous hemofiltration using a bicarbonate buffered, calcium containing replacement solution. Blood Purif. 2016; 42(4): 349-355, doi: 10.1159/000452755, indexed in Pubmed: 27866200.

11. Passaglia LG, de Barros GM, de Sousa MR. Early postoperative bridging anticoagulation after mechanical heart valve replacement: a systematic review and meta-analysis. J Thromb Haemost. 2015; 13(9): 1557-1567, doi: 10.1111/jth.13047, indexed in Pubmed: 26178802.

12. Giani M, Scaravilli V, Stefanini F, et al. Continuous renal replacement therapy in venovenous extracorporeal membrane oxygenation: a retrospective study on regional citrate anticoagulation. ASAIO J. 2020; 66(3): 332-338, doi: 10.1097/ MAT.0000000000001003, indexed in Pubmed: 31045918.

13. Zwicker JI, Uhl L, Huang WY, et al. Thrombosis and ELISA optical density values in hospitalized patients with heparin-induced thrombocytopenia. J Thromb Haemost. 2004; 2(12): 2133-2137, doi: 10.1111/j.1538-7836.2004.01039.x, indexed in Pubmed: 15613017.

14. Morabito S, Pistolesi V, Tritapepe L, et al. Regional citrate anticoagulation in cardiac surgery patients at high risk of bleeding: a continuous veno-venous hemofiltration protocol with a low concentration citrate solution. Crit Care. 2012; 16(3): R111, doi: 10.1186/cc11403, indexed in Pubmed: 22738289.

15. Huguet M, Rodas L, Blasco M, et al. Clinical impact of regional citrate anticoagulation in continuous renal replacement therapy in critically ill patients. Int J Artif Organs. 2017; 40(12): 676-682, doi: 10.5301/ijao.5000633, indexed in Pubmed: 28862718.

16. Zuščich O, Hájek R, Ševčík P, et al. The effect of continuous elimination methods on the hemostatic profile of a cardiac surgery patient monitored using thromboelastography. Int J Artif Organs. 2016; 39(3): 106-113, doi: 10.5301/ijao.5000490, indexed in Pubmed: 27102694.

17. De Vico P, Messino V, Tartaglione A, et al. Safety and efficacy of citrate anti-coagulation continuous renal replacement therapies in post-cardiac surgery patients with liver dysfunction. Ther Apher Dial. 2015; 19(3): 272-278, doi: 10.1111/1744-9987.12280, indexed in Pubmed: 25656632.

18. Gattas DJ, Rajbhandari D, Bradford C, et al. A randomized controlled trial of regional citrate versus regional heparin anticoagulation for continuous renal replacement therapy in critically ill adults. Crit Care Med. 2015; 43(8): 1622-1629, doi: 10.1097/ CCM.0000000000001004, indexed in Pubmed: 25853591.

19. Schilder L, Nurmohamed SA, Bosch FH, et al. CASH study group. Citrate anticoagulation versus systemic heparinisation in continuous venovenous hemofiltration in critically ill patients with acute kidney injury: a multi-center randomized clinical trial. Crit Care. 2014; 18(4): 472, doi: 10.1186/s13054-014-0472-6, indexed in Pubmed: 25128022. 
20. Morgera S, Schneider M, Slowinski T, et al. A safe citrate anticoagulation protocol with variable treatment efficacy and excellent control of the acid-base status. Crit Care Med. 2009; 37(6): 2018-2024, doi: 10.1097/CCM.0b013e3181a00a92, indexed in Pubmed: 19384210.

21. Wu B, Zhang K, Xu B, et al. Randomized controlled trial to evaluate regional citrate anticoagulation plus low-dose of dalteparin in continuous veno-venous hemofiltration. Blood Purif. 2015; 39(4): 306-312, doi: 10.1159/000381662, indexed in Pubmed: 26022434.

22. Kirwan CJ, Jackson L, Prowle JR. A continuous renal replacement therapy protocol on the updated nikkiso aquarius platform using regional citrate as first-line anticoagulation significantly improves filter life span but the position of the vascular access is key. Blood Purif. 2018; 45(1-3): 129-130, doi: 10.1159/000485232, indexed in Pubmed: 29402829.

23. Squiccimarro E, Labriola C, Malvindi PG, et al. Prevalence and clinical impact of systemic inflammatory reaction after cardiac surgery. J Cardiothorac Vasc Anesth. 2019; 33(6): 1682-1690, doi: 10.1053/j.jvca.2019.01.043, indexed in Pubmed: 30772177.

24. Kellum JA, Johnson JP, Kramer D, et al. Diffusive vs. convective therapy: effects on mediators of inflammation in patient with severe systemic inflammatory response syndrome. Crit Care Med. 1998; 26(12): 1995-2000, doi: 10.1097/00003246-19981200000027, indexed in Pubmed: 9875910.
25. Moledina DG, Isguven S, McArthur E, et al. Translational Research Investigating Biomarker Endpoints in Acute Kidney Injury (TRIBE-AKI) Consortium. Plasma Monocyte Chemotactic Protein-1 Is Associated With Acute Kidney Injury and Death After Cardiac Operations. Ann Thorac Surg. 2017; 104(2): 613-620, doi: 10.1016/j.athoracsur.2016.11.036, indexed in Pubmed: 28223055.

26. Clementi A, Brocca A, Virzì GM, et al. Procalcitonin and interleukin-6 levels: are they useful biomarkers in cardiac surgery patients? Blood Purif. 2017; 43(4): 290-297, doi: 10.1159/000454672, indexed in Pubmed: 28125806.

27. Schilder L, Nurmohamed SA, ter Wee PM, et al. Citrate confers less filter-induced complement activation and neutrophil degranulation than heparin when used for anticoagulation during continuous venovenous haemofiltration in critically ill patients. BMC Nephrol. 2014; 15: 19, doi: 10.1186/1471-2369-15-19, indexed in Pubmed: 24438360.

28. Bienholz A, Reis J, Sanli P, et al. Citrate shows protective effects on cardiovascular and renal function in ischemia-induced acute kidney injury. BMC Nephrol. 2017; 18(1): 130, doi: 10.1186/ s12882-017-0546-1, indexed in Pubmed: 28395656.

29. Oudemans-van Straaten HM, Bosman RJ, Koopmans M, et al. Citrate anticoagulation for continuous venovenous hemofiltration. Crit Care Med. 2009; 37(2): 545-552, doi: 10.1097/ CCM.0b013e3181953c5e, indexed in Pubmed: 19114912. 